\title{
La enseñanza de la pronunciación en los manuales de ELE: evolución y estado actual
}

\author{
Pronunciation teaching in SFL textbooks: evolution and current \\ state
}

ALBA IGARRETA FERNÁNDEZ

PROFESORA AUTÓNOMA DE LENGUA EXTRANJERA

alba.igarreta@gmail.com

\begin{abstract}
Resumen
El tratamiento de la pronunciación ha variado según las metodologías de enseñanza de lenguas extranjeras empleadas a lo largo de la historia, aspecto que se ha visto reflejado, tanto en el aula, como en los manuales de ELE. Este artículo analiza dichas metodologías, las obras de referencia para la creación y diseño de materiales didácticos y de cursos, la inclusión de la pronunciación en los manuales de ELE, y propuestas para integrar su enseñanza en el aula. Esto permite conocer la evolución y el estado actual de la enseñanza de la pronunciación con el fin de mejorarla.

Palabras clave: enseñanza de la pronunciación, manual de ELE, corrección fonética, currículo, metodología
\end{abstract}

\begin{abstract}
The treatment of pronunciation has varied depending on the foreign language teaching methodologies used throughout history, an aspect that has been reflected both in the classroom and in SFL textbooks. This article analyzes these methodologies, the reference works for the creation and design of teaching materials and courses, the inclusion of pronunciation in SFL textbooks, and proposals to integrate their teaching in the classroom. This allows to know the evolution and the current state of the pronunciation teaching to improve it.

Keywords: pronunciation teaching, SFL textbook, phonetic correction, curriculum, methodology
\end{abstract}




\section{Introducción}

La importancia que se ha atribuido a la pronunciación en las clases de lenguas extranjeras ha variado a lo largo de la historia, especialmente en función de las corrientes metodológicas que predominaban en diferentes momentos de la historia (Diccionario de términos clave de ELE: 2008). Como se verá más adelante en este trabajo, la pronunciación ha alcanzado gran relevancia en el proceso de enseñanza-aprendizaje de lenguas en determinados momentos, mientras que en otros su papel ha sido meramente anecdótico.

También existe una creencia generalizada entre el profesorado de español como lengua extranjera según la cual los manuales de ELE suelen dejar de lado los aspectos relacionados con la pronunciación de la lengua, como indican Casany y Landa (2010: 1). Por este motivo, en este trabajo también se analizan los resultados extraídos de diferentes investigaciones que estudian la inclusión de actividades de pronunciación en diversos manuales de ELE.

Cabe señalar que, tal y como apunta Bartolí Rigol (2005), a lo largo de la historia los investigadores han ofrecido diferentes definiciones relativas a la pronunciación. Llisterri (2003) señala que algunos conceptos como "enseñanza de la fonética", "enseñanza de la pronunciación" y "corrección fonética" suelen ser confundidos y mal interpretados. En este trabajo se emplean estos términos según la definición de estos que propone el investigador (Llisterri, 2003: 91-92):

- Enseñanza de la fonética: reflexión explícita sobre el sistema que suele realizarse en el marco de los estudios de Filología, con la que se adquiere conocimiento formal y detallado de las características articulatorias, acústicas y perceptivas de los elementos segmentales y suprasegmentales de la lengua.

- Enseñanza de la pronunciación: la pronunciación es una de las destrezas que los estudiantes de una lengua extranjera deben dominar, que debe formar parte del contenido curricular, y que los docentes deben incorporar en las actividades de clase.

- Corrección fonética: es necesaria cuando en la producción oral se detectan errores que es preciso corregir. Para ello, existen estrategias y procedimientos que el docente debe conocer.

Este artículo se centra en la enseñanza de la pronunciación y la corrección fonética, y en su presencia en los manuales de ELE.

Iruela (2004) señala que "la pronunciación es el soporte de la transmisión de la información oral" y su importancia comunicativa "reside en que otorga inteligibilidad al texto oral" (Iruela, 2004: 28). Bartolí Rigol (2005: 3) añade que "la pronunciación no es solo producción sino también la percepción de los sonidos del habla". Trabajos como los de Gospodaric (2004) e Igarreta Fernández (2015) demuestran que recibir instrucción en 
pronunciación ayuda a disminuir la percepción errónea y a mejorar la producción.

Llegados a este punto, resulta cuanto menos curioso que, si bien parece haber consenso en torno a la importancia de incluir la pronunciación en el proceso de enseñanza y aprendizaje de ELE, esta no se incluye en la actividad docente de forma regular. Esto se debe, en parte, a que existen ciertos prejuicios y creencias que pueden influir en que en la práctica docente cotidiana se preste poca atención a la enseñanza de la pronunciación. De acuerdo con Poch (2004),

dos son los factores que explicarían esta situación: en primer lugar la existencia de una serie de prejuicios sobre los aspectos fonéticos o de pronunciación del español y, en segundo lugar, una creencia muy extendida de que para abordar y tratar las cuestiones de pronunciación hay que ser especialista en fonética y no es ésta la formación que poseen habitualmente los profesores de español lengua extranjera. (Poch, 2004: 1).

Orta Gracia (2009) lleva a cabo un estudio en el que analiza dichas creencias y sus repercusiones en el aula. Los docentes que forman parte de la investigación piensan que la enseñanza de la pronunciación no tiene la misma relevancia que la enseñanza de la gramática, o que el sistema fonético del español es más sencillo que el de otras lenguas, por lo que el estudiante puede adquirirlo de forma autodidacta. Como consecuencia de estas ideas, los profesores dedican menos tiempo, si lo hacen, a la enseñanza de la pronunciación que a la de contenidos relacionados con la gramática y el léxico.

Los docentes que deciden integrar la enseñanza de la pronunciación en su programación se pueden encontrar con diversas dificultades, ya que, como indica Gil Fernández (2007: 126): "en la actualidad no se cuenta con un marco teórico suficientemente claro y probado, a la par que bien difundido entre los docentes, que pueda funcionar como referente para enfocar y abordar las clases". Sin embargo, esto no siempre ha sido así, por lo que en el siguiente apartado se realiza un breve repaso de las diferentes metodologías de enseñanza de segundas lenguas para conocer cuál ha sido la evolución de la enseñanza de la pronunciación a lo largo de los años y, de esta manera, conocer las razones por las que en la actualidad es tratada como se ha indicado en estas líneas.

\section{La pronunciación en las diferentes metodologías de enseñanza de lenguas extranjeras}

Como se ha dicho, existen diferentes teorías, métodos y enfoques relacionados con la enseñanza de segundas lenguas y de lenguas extranjeras que a lo largo de la historia han tratado de forma diferente la cuestión de la pronunciación. 
A finales del siglo XIX y comienzos del XX el objetivo de renombrados fonetistas como Henry Sweet o Paul Passy era mejorar la enseñanza de idiomas. Tal y como indica Gil Fernández (2007), sus trabajos mostraron la necesidad de aplicar a la enseñanza y a la formación de profesores los hallazgos de la investigación en fonética. Además, Gil Fernández (2007) apunta que

(e)llos fueron los auténticos precursores de la manera actual de enseñar pronunciación y, para que su aportación fuera definitiva, sólo les faltó que su enfoque se concretara en un auténtico método, un plan detallado y estructurado con procedimientos específicos para poner en práctica en el aula los principios generales de los que partían (Gil Fernández, 2007: 128).

Es posible que, debido a esa falta de concreción y método, la pronunciación haya sido tratada de diversas maneras en los diferentes métodos y enfoques de enseñanza de lenguas. La tabla 1 muestra, de manera resumida, dicho tratamiento. Esta tabla está basada en el Diccionario de términos clave de ELE (2008) y en los trabajos de Renard (1979), Bartolí Rigol (2005), Gil Fernández (2007), Orta Gracia (2009), Tiedong Yang (2013), Igarreta Fernández (2015), González Sánchez (2016) y Torrero Díaz (2020).

Tabla 1. Tratamiento de la pronunciación según los diferentes métodos y enfoques de enseñanza de segundas lenguas o de lenguas extranjeras

\begin{tabular}{|c|c|}
\hline $\begin{array}{l}\text { Método o } \\
\text { Enfoque }\end{array}$ & Tratamiento de la pronunciación y su enseñanza \\
\hline $\begin{array}{l}\text { Método } \\
\text { gramática- } \\
\text { traducción }\end{array}$ & $\begin{array}{l}\text { - La pronunciación no se tiene en cuenta, es relegada a último } \\
\text { plano }\end{array}$ \\
\hline Método directo & $\begin{array}{l}\text { - La pronunciación se enseña mediante la imitación y la } \\
\text { repetición de pequeños diálogos situacionales } \\
\text { - La corrección es prioritaria }\end{array}$ \\
\hline $\begin{array}{l}\text { Método fono- } \\
\text { articulatorio }\end{array}$ & $\begin{array}{l}\text { - Enseñanza deductiva de la pronunciación } \\
\text { - El docente presenta el sistema fonético de la lengua meta e } \\
\text { invita a contrastarlo con el de la L1 } \\
\text { - Adquisición consciente de la pronunciación: palpación de los } \\
\text { movimientos del aparato fonador durante la producción del } \\
\text { - habla, pronunciación con los ojos cerrados y en voz baja } \\
\text { - La percepción y distinción de fonemas queda relegada a un } \\
\text { - Segundo plano }\end{array}$ \\
\hline $\begin{array}{l}\text { Método } \\
\text { audiolingüistico }\end{array}$ & $\begin{array}{l}\text { - La pronunciación desempeña un papel principal } \\
\text { - La corrección es prioritaria } \\
\text { - El estudiante es capaz de adquirir los nuevos elementos } \\
\text { fónicos de manera inductiva partiendo del uso y de la } \\
\text { repetición de ejemplos } \\
\text { - El objetivo principal es la formación de hábitos fónicos: } \\
\text { automatizar la percepción auditiva y la producción } \\
\text { - El profesor presenta listas de pares mínimos, aislados o en } \\
\text { oraciones, e induce a los estudiantes a discriminar } \\
\text { auditivamente los fonemas para después repetirlos }\end{array}$ \\
\hline
\end{tabular}




\begin{tabular}{|c|c|}
\hline Enfoque natural & $\begin{array}{l}\text { - Se supone que la pronunciación se aprende de forma } \\
\text { inconsciente, sin tratamiento explícito, a través de la } \\
\text { exposición a textos orales } \\
\text { - El alumno tiene que escuchar y, con el tiempo, producir } \\
\text { - Alta tolerancia frente a los errores de pronunciación }\end{array}$ \\
\hline $\begin{array}{l}\text { Método respuesta } \\
\text { física total }\end{array}$ & $\begin{array}{l}\text { - Se centra en la recepción de los sonidos; el alumno escucha } \\
\text { más de lo que habla } \\
\text { - Alta tolerancia frente a los errores de pronunciación }\end{array}$ \\
\hline Método silencioso & $\begin{array}{l}\text { - La producción oral se centra en la gramática } \\
\text { - La fluidez es prioritaria } \\
\text { - El alumno debe deducir la pronunciación correcta }\end{array}$ \\
\hline Sugestopedia & $\begin{array}{l}\text { - La producción oral es muy controlada } \\
\text { - La fluidez es prioritaria } \\
\text { - Se leen diálogos con variación entonativa }\end{array}$ \\
\hline $\begin{array}{l}\text { Aprendizaje de la } \\
\text { lengua en } \\
\text { comunidad }\end{array}$ & $\begin{array}{l}\text { - Actividades de repetición } \\
\text { - La fluidez es prioritaria }\end{array}$ \\
\hline $\begin{array}{c}\text { Enfoque } \\
\text { comunicativo }\end{array}$ & $\begin{array}{l}\text { - La fluidez y la eficacia comunicativa son prioritarias } \\
\text { - Se supone que la pronunciación está integrada en el resto } \\
\text { de las competencias orales } \\
\text { - La inteligibilidad y la fluidez son prioritarias para garantizar } \\
\text { la comunicación } \\
\text { - El "acento extranjero" se tolera siempre y cuando no impida } \\
\text { la comunicación. Lograr un acento nativo se considera un } \\
\text { objetivo poco realista }\end{array}$ \\
\hline $\begin{array}{l}\text { Enfoque por } \\
\text { tareas }\end{array}$ & $\begin{array}{l}\text { - La fluidez y la eficacia comunicativa son prioritarias y se } \\
\text { anteponen a la corrección } \\
\text { - La pronunciación está integrada en los procesos de } \\
\text { comunicación oral }\end{array}$ \\
\hline $\begin{array}{c}\text { Método verbo- } \\
\text { tonal }\end{array}$ & $\begin{array}{l}\text { - El estudiante de una lengua extranjera tiene una sordera } \\
\text { fonológica en lo que respecta a los sonidos de dicha lengua } \\
\text { - La corrección fonética se debe iniciar desde la percepción } \\
\text { - En la fase de producción, el estudiante realiza ejercicios de } \\
\text { pronunciación matizada y fonética combinatoria }\end{array}$ \\
\hline
\end{tabular}

Si bien a lo largo de la historia la tendencia general ha sido la de adoptar un método y ceñirse a sus características, en la actualidad la tendencia es a seguir un enfoque ecléctico, ya que, en la que algunos autores denominan la etapa posmétodo (Kumaravadivelu, 1994), se combinan principios de diferentes metodologías. Por este motivo, en dicho enfoque no se sigue una teoría específica respecto a la pronunciación.

Tras haber expuesto los aspectos relacionados con el tratamiento de la pronunciación en los distintos métodos, resulta necesario analizar cómo es tratada en las obras curriculares de referencia.

\section{La pronunciación en el currículo}

En la actualidad, tanto editoriales como organizadores de cursos de formación de profesores, y los propios docentes, emplean obras de referencia que sirven como guía para la elaboración de programas de 
lenguas, currículos, o manuales, entre otros. En este apartado se analizan brevemente dos de ellas: el Marco Común Europeo de Referencia para las Lenguas (MCER, en adelante) y el Plan curricular del Instituto Cervantes (PCIC, en adelante).

\subsection{Marco Común Europeo de Referencia para las Lenguas}

EI MCER divide las competencias comunicativas de la lengua en competencias lingüísticas, sociolingüísticas y pragmáticas. La pronunciación constituye una de las competencias lingüísticas, dentro de la cual se distinguen la competencia ortoépica y la fonológica. La primera está relacionada con "el conocimiento y la destreza en la percepción y la producción de los símbolos de que se componen los textos escritos", mientras que la segunda supone el conocimiento y la destreza en la percepción y la producción de los sonidos, los rasgos fonéticos, la composición y reducción fonética, y la fonética de las oraciones (MCER, 2002: 113-114).

En cuanto a los seis niveles de competencia fónica que suponen el dominio de la pronunciación, son descritos desde un punto de vista de la producción, y no de la percepción. En los niveles $A 1, A 2$ y B1 se asume como normal que los estudiantes tengan "acento extranjero", y solo se mencionan aspectos suprasegmentales, como la entonación, a partir del nivel B2, nivel en el que la pronunciación del estudiante debería ser clara y natural (MCER: 2012: 114).

EI MCER, que en general tiene un enfoque comunicativo, indica que se espera que los alumnos desarrollen su capacidad de pronunciar una lengua mediante la exposición a enunciados auténticos, por imitación a coro del profesor o de grabaciones de hablantes nativos, mediante el trabajo individual en el laboratorio de idiomas, leyendo en alto material textual fonéticamente significativo, mediante el entrenamiento auditivo y con ejercicios fonéticos de repetición, con el uso de textos transcritos fonéticamente, mediante el entrenamiento fonético explícito, aprendiendo las normas ortoépicas, o mediante alguna combinación de las anteriores (MCER, 2002: 152).

En cuanto a la implementación de lo indicado en el MCER, el mismo documento indica que "no nos proponemos decir a los profesionales lo que tienen que hacer o de qué forma hacerlo" (MCER, 2002: XI), y señala que es tarea de los usuarios determinar qué destrezas fonológicas nuevas se le piden al alumno, cuál es la importancia relativa de los sonidos y de la prosodia, y si la corrección y la fluidez fonética son un objetivo temprano del aprendizaje o si se desarrollan como un objetivo a largo plazo (MCER, 2002: 114). 


\subsection{Plan curricular del Instituto Cervantes}

El Plan curricular del Instituto Cervantes - Niveles de referencia (2006) desarrolla los objetivos y contenidos para cada nivel de enseñanza del español como lengua extranjera a partir de las directrices del MCER.

En lo que concierne a la metodología, el PCIC indica que la mayor parte de los especialistas en didáctica de la pronunciación de segundas lenguas "propugna en la actualidad una orientación ecléctica y de naturaleza integradora, en la que eventualmente tengan cabida las aportaciones de cada uno de los modelos existentes que se hayan demostrado positivas" (PCIC, 2006). Por lo tanto, si bien no propone una metodología específica, indica que el docente debe estar preparado académicamente para poder adaptarse a las necesidades de los estudiantes en cada momento, y debe ser capaz de integrar las actividades de corrección fonética en el conjunto de actividades que se llevan a cabo en el aula. Además, el PCIC recalca que la enseñanza de la pronunciación debe basarse en la interacción comunicativa, y no en la práctica repetitiva de las formas aisladas.

En cuanto a la ordenación jerárquica de los distintos aspectos fonéticos, el PCIC parte de que la base es la articulación general, y ordena el plano suprasegmental en primer lugar, dado que reviste una gran importancia para la comunicación. En segundo lugar, sitúa los aspectos segmentales. Esto contrasta con lo indicado en el MCER, ya que, según este, el grado de dominio de la lengua en niveles inferiores se centra en aspectos segmentales, y es a partir del nivel B2 cuando se incluyen los aspectos suprasegmentales.

EI PCIC agrupa los diferentes niveles de referencia en tres fases e indica cuáles son los objetivos en el campo de la pronunciación de cada una de ellas. El de la fase de aproximación (niveles A1 y A2) es reconocer los patrones fónicos del español y reproducir sus esquemas básicos. La meta de la fase de profundización (niveles B1 y B2) es que el estudiante se aproxime más a la base articulatoria del español, que pueda pronunciar correctamente las secuencias vocálicas y consonánticas, tanto en el interior de una palabra como en enunciados, y que sea capaz de expresar determinados estados emocionales a través de la pronunciación. En cuanto a la fase de perfeccionamiento (niveles C1 y C2), el objetivo es que la pronunciación del estudiante sea muy similar a la de un hablante nativo, que sea capaz de tener en cuenta la adecuación a los estados de ánimo y a las intenciones pragmáticas, y que pueda modificar el tempo y la articulación conforme a la situación comunicativa y el registro.

Como se ha podido observar en este apartado, si bien las obras de referencia analizadas ofrecen una serie de indicaciones y pautas en lo que a la enseñanza de la pronunciación se refiere, no presentan una línea metodológica o un enfoque didáctico definido. De hecho, ambas indican que las nociones que proporcionan permiten adaptarlas a lo que los usuarios consideren más relevante en relación con la didáctica de la pronunciación. 
Puesto que estas son las obras de referencia a la hora de crear materiales didácticos, en el siguiente apartado se analiza el tratamiento de la pronunciación en los manuales de ELE.

\section{La enseñanza de la pronunciación en los manuales de ELE}

Como se ha comentado en el apartado de introducción, en la actualidad tanto profesores de ELE como investigadores señalan que, tanto en la enseñanza de ELE, como en los manuales, "la pronunciación sigue estando desatendida y que no recibe el tratamiento adecuado" (Bartolí Rigol: 2005: 3). Además, en el caso de los materiales didácticos se puede observar que "la introducción a los sonidos de la LE y la gran mayoría de actividades de pronunciación propuestas en ellos se supeditan a la escritura" (Bartolí Rigol, 2005: 8). Según apuntan Casany y Landa,

(e)n los manuales de ELE solo hay, comúnmente, un único tema dedicado a la pronunciación, que suele centrarse en las consonantes, las vocales y las combinaciones de ambas, dejando de lado temas tan importantes como la acentuación y la prosodia, imprescindibles para la correcta pronunciación del español. (Casany y Landa: 2010: 1).

No obstante, no hay dudas respecto a la utilidad y los beneficios del empleo de manuales en el aula, puesto que, como indica Ezeiza (2009: 16), "las voces más autorizadas en los ámbitos de la didáctica de lenguas, la formación de profesores y el desarrollo de políticas lingüísticas" coinciden plenamente en que estos materiales "cumplen diversas e importantes funciones en los procesos de enseñanza-aprendizaje". Núñez Delgado y Rodríguez Guerrero (2017) subrayan la relevancia de estos, e indican que "(e)l manual o libro de texto siempre será una herramienta primordial de trabajo a la que, tanto profesor como alumno, pueden recurrir y ofrecerá los recursos necesarios para contribuir al aprendizaje significativo del alumno, en todas y cada una de las competencias" (Núñez Delgado y Rodríguez Guerrero, 2017: 38). La relevancia de estos materiales y el uso generalizado de los mismos hacen que resulte necesario examinar los manuales para conocer más detalladamente aspectos como la manera en la que se introduce la pronunciación en los mismos, si se incluye de manera sistemática en todas las unidades, o la tipología de actividades que se incluyen para trabajarla.

El análisis de manuales de español "cuenta con una trayectoria ciertamente consolidada", como indica González Sánchez (2016: 35). Uno de los pioneros en este campo fue Ezquerra (1974), quien, basándose en los diferentes métodos de enseñanza de lenguas creó un esquema en el que incluyó los principales parámetros a seguir a la hora de llevar a cabo el estudio de manuales. El investigador sienta las bases para analizar los métodos de la manera más objetiva y explícita posible, de forma que sea posible comparar unos manuales con otros, incluso si corresponden a concepciones teóricas y prácticas diferentes (Ezquerra, 1974: 46). 
Si bien es cierto que existe una amplia bibliografía que examina los manuales de español, no se encuentran muchos trabajos en los que se analice en detalle el tratamiento de la pronunciación en los mismos, a pesar de que Ezquerra sí lo incluya en su trabajo. El investigador realiza diferentes observaciones respecto a lo que denomina el contenido fonético, las cuales resultan sumamente interesantes ya que permiten conocer cuál era la situación de la pronunciación en los métodos empleados en la década de los 70, y cómo ha sido la evolución de estos. Ezquerra (1974: 36) observa que "(e)n general, desde la primera o primeras lecciones en los métodos existentes se introducen todos los elementos fonéticos y fonológicos de forma totalmente inorganizada". El autor indica que en el Centro de Lingüística Aplicada de Besançon dedicaban cinco horas a la sensibilización fonética y fonológica de los estudiantes en una fase previa a iniciar la enseñanza de la lengua, y que en el momento de desarrollar su investigación estaban en fase de proyecto los ejercicios posteriores de corrección fonética. Estos ejercicios específicos y pioneros no aparecían en los diferentes métodos de la época y, según Ezquerra, "todo método de español debería de acompañarse de este tipo de ejercicios" (Ezquerra, 1974: 36).

Bartolí Rigol (2012) desarrolla una tesis doctoral en la que realiza un análisis detallado de 44 manuales de diferente tipo. De estos, 29 de han sido concebidos para la enseñanza y aprendizaje de ELE. La investigadora indica que únicamente 9 contemplan la pronunciación en su tabla de contenidos, y el resto no presentan contenidos de pronunciación de forma sistemática. De estos últimos manuales, algunos no presentan referencias a este aspecto de la lengua, otros se limitan a la presentación de los sonidos según su correspondencia ortográfica, otros contienen referencias en algunas unidades o le dedican un anexo, y otros ofrecen actividades de pronunciación en el cuaderno de ejercicios (Bartolí Rigol, 2012: 191).

Por un lado, Bartolí Rigol (2012: 440-441) observa que, en general, la pronunciación se suele trabajar desvinculada de las actividades orales y relacionada con las reglas ortográficas, y solo en algunos manuales se conecta con la gramática y con la adquisición del léxico. También señala que, en general, las actividades "consisten en escuchar, repetir, clasificar, discriminar, leer y/o completar textos" (Bartolí Rigol, 2012: 440). Además, generalmente se trabajan los sonidos aislados, en palabras y frases, y son pocos los manuales que incorporan la práctica de los elementos suprasegmentales, y normalmente lo hacen con mediación de la lengua escrita.

Por otro lado, Bartolí Rigol (2012) analiza los contenidos de pronunciación a nivel curricular y observa que la adecuación "a las directrices del MCER y al antiguo o al nuevo Plan curricular no depende del año de publicación del manual" (Bartolí Rigol, 2012: 449). En cuanto al orden de introducción de los temas en los métodos que contienen sección de pronunciación, Bartolí Rigol (2012: 449), indica que ninguno de los analizados sigue el orden propuesto por el PCIC, el cual, como se ha indicado en el anterior apartado, ordena en primer plano el plano suprasegmental, y en segundo el segmental. 
González Sánchez (2016) toma como base las aportaciones de Ezeiza (2009) y realiza un análisis metodológico de nueve manuales que siguen tres vertientes metodológicas: el enfoque por tareas, el enfoque orientado a la acción y el eclecticismo (González Sánchez, 2016: 46). La autora analiza si la pronunciación se trabaja y de qué modo, y qué lugar ocupan en la unidad las actividades diseñadas para tal fin, y concluye que "la pronunciación sigue siendo la gran olvidada en muchos de los manuales analizados" (González Sánchez, 2016: 111). Además, añade que muchos de ellos incluyen pocas actividades, o incluso se limitan a "aconsejar al alumno que cuide este aspecto cuando se exprese oralmente" (González Sánchez, 2016: 111).

En relación con la metodología que siguen los manuales, González Sánchez (2016) indica que "en muchos de ellos, a pesar de defender un método concreto de gran actualidad, se hallan reminiscencias a enfoques metodológicos anteriores" y que, además, "en ciertas ocasiones los autores afirman adoptar algunas medidas que, finalmente, no se perciben en la práctica" (González Sánchez, 2016: 116-117).

El trabajo de Šifrar Kalan (2017), se centra en la enseñanza de la pronunciación en tres manuales de ELE que, como indica la autora, siguen un enfoque comunicativo ecléctico. En primer lugar, analiza si siguen una enseñanza sistemática de la pronunciación por unidades y niveles, y señala que solo uno lo hace, en otro aparecen ejercicios de forma esporádica, y en otro, según aumenta el nivel el número de ejercicios para trabajar la competencia fonológica es cada vez menor (Šifrar Kalan, 2017: 324-325). También analiza el orden en el que se presentan los elementos segmentales y suprasegmentales y observa que, en general, empiezan por los sonidos consonánticos y vocálicos, se invita al estudiante a observar la diferencia entre el español y su lengua materna, y también en algunos casos la diferencia entre variedades del español. En cuanto a los elementos suprasegmentales, "no se enseñan de manera sistemática ni acorde con la teoría de adquisición que aboga por una enseñanza temprana de la prosodia ni con las sugerencias de los expertos" (Šifrar Kalan, 2017: 325). En cuanto a la tipología de actividades, Šifrar Kalan (2017: 325) indica que, en general, "no se apartan de los métodos tradicionales intuitivo-imitativos", ya que la percepción se basa en actividades de escuchar y observar, y la producción en mera reproducción.

Cabe señalar que, si bien el último trabajo expuesto en este artículo data del año 2017, posteriormente diversas editoriales han publicado manuales de ELE, por lo que resultaría necesario analizar los mismos como se ha hecho con los métodos publicados con anterioridad.

Los trabajos expuestos en este apartado permiten llegar a diferentes conclusiones. Por un lado, parece que, generalmente, los manuales de ELE siguen una metodología, en cierta manera, ecléctica, ya que, si bien indican seguir un enfoque específico, las investigaciones muestran que en ellos se encuentran rasgos de enfoques anteriores.

Por otro lado, los elementos segmentales y suprasegmentales se presentan siguiendo un orden que difiere del indicado en el PCIC, ya que la 
tendencia general es presentar, en primer lugar, los elementos segmentales, y en niveles más avanzados, los suprasegmentales.

En cuanto a las competencias fonológica y ortoépica, a pesar de que el MCER distingue claramente ambas, se ha comprobado que, generalmente, la pronunciación se trabaja desvinculada de las actividades orales, y que suele relacionarse con la competencia ortoépica.

En relación con el tipo de actividades, la tendencia general es incluir actividades que consisten en escuchar, repetir, clasificar, discriminar, leer y/o completar textos, suelen encontrarse en apartados sobre pronunciación y ortografía o en el cuaderno de ejercicios, y, si bien sirven para conocer las diferencias entre los sonidos de la L1 y la lengua extranjera, no garantizan la adquisición de la competencia fónica.

\section{Propuestas para integrar la enseñanza de la pronunciación en el aula de ELE}

Poch y Harmegies (2009) consideran que, en lo que concierne a la didáctica de la lengua extranjera, hay dos tareas fundamentales previas a la labor que se desarrolla en el aula. La primera de ellas es definir los contenidos que se van a enseñar. La segunda consiste en "diseñar la metodología que permitirá la transmisión de estos contenidos de la forma más eficaz posible" (Poch y Harmegies, 2009: 108). Como se ha visto en anteriores apartados, los docentes, en general, definen los contenidos siguiendo los manuales de ELE, los cuales, a su vez, han sido diseñados siguiendo las obras de referencia, como el MCER y el PCIC. Sin embargo, se ha observado que no existe consenso en lo que a la metodología para la enseñanza de la pronunciación se refiere.

Investigadores como Bartolí Rigol (2005) señalan la necesidad de crear y desarrollar nuevos materiales didácticos y actividades de aprendizaje. Con este fin, en los últimos años se han publicado trabajos en los que se realizan propuestas para incluir e integrar la pronunciación en el aula de ELE, tanto de forma general, como aspectos concretos de la misma.

Bartolí Rigol (2012) elabora una propuesta didáctica en la que se integra la enseñanza de la pronunciación en la enseñanza de la lengua oral, aplicando para ello el método didáctico del enfoque por tareas. De acuerdo con la autora,

(i)ntegrar la enseñanza de la pronunciación en la práctica de la lengua oral significa (...) practicar contenidos de pronunciación y funciones lingüísticas simultáneamente, en actividades orales basadas en la interacción, en las que el apoyo de la lengua escrita o en las que su uso sea mínimo. (Bartolí Rigol, 2012: 452).

Esta propuesta se puede adaptar a cualquier programa o manual, y puede convertirse en un complemento de estos. Además, Bartolí Rigol (2012) defiende que mediante el enfoque por tareas "es posible introducir en el aula tareas derivadas de naturaleza tradicional, es decir, prácticas centradas 
en la percepción y la producción, con uso de métodos fonoarticulatorios y destinadas a la clásica relación sonido y grafía, sin que ello suponga un añadido desconectado del resto" (Bartolí Rigol, 2012: 453).

El trabajo de Molina Vidal (2014) se centra especialmente en la enseñanza de la entonación, y propone diferentes actividades que divide en tres etapas. La primera es la de la presentación de un modelo para que el estudiante reflexione sobre el mismo, la explicación del docente, la representación visual, la contextualización y la ejemplificación. La segunda etapa es la de la ejercitación, en la que se realizan actividades de imitación, memorización, discriminación, transformación de un modelo, sistematización, y práctica en parejas o en grupos. La tercera etapa es la de supervisión, es decir, la corrección y la evaluación.

El trabajo de fonética aplicada de Igarreta Fernández (2015) muestra cómo se puede acerca la pronunciación de un grupo de estudiantes sinohablantes a la de los hispanohablantes nativos. Tras analizar la producción del grupo de informantes, la investigadora diseña una secuencia didáctica basada en los principios del método verbo-tonal. En primer lugar, se realizan actividades de discriminación auditiva en palabras aisladas; en segundo lugar, actividades de discriminación auditiva en oraciones; en tercer lugar, se realizan ejercicios de pronunciación matizada; en cuarto lugar, se llevan a cabo ejercicios de repetición de palabras descontextualizadas; por último, se realizan ejercicios de fonética combinatoria. Los resultados de este trabajo permiten suponer que la incorporación de las técnicas propias del método verbo-tonal a las clases regulares de ELE permitiría acercar la pronunciación del español por sinohablantes, o por hablantes de otras lenguas, a la de los nativos.

El trabajo de Pérez Cano (2019) se centra en la conciencia fonológica de los estudiantes de ELE, y propone una serie de tareas para trabajarla como recurso didáctico de comprensión auditiva. Dichas tareas se pueden clasificar en tres clases: sensibilidad fonológica superficial, media y profunda. La sensibilidad fonológica superficial consiste en escuchar y repetir logotomas o palabras reales, elegir de entre un par o un trío de palabras cuál no pertenece al grupo, y reconocer una rima. La sensibilidad fonológica media consiste en silabear y en contar y fusionar fonemas. La sensibilidad fonológica profunda consiste en reconocer el fonema inicial y unirlo con otras palabras, operar con el estímulo presentado identificando un fonema concreto, repetir el impulso quitándole un fonema y suprimir el fonema inicial (Pérez Cano, 2019: 73).

Torreo Díaz (2020) propone un modelo de secuenciación didáctica cuyo objetivo es facilitar la inclusión de la enseñanza de la entonación en el sílabo de cualquier curso. La autora tiene en cuenta "las metodologías anteriores y las críticas que han recibido para poder ofrecer un modelo que intente suplir esas carencias y que se pueda adaptar a las necesidades del grupo de alumnos con el que nos encontremos" (Torrero Díaz, 2020: 1). La secuencia propuesta consta de cinco niveles: presentación, ilustración, percepción, ejercitación y producción libre. 
En este apartado se ha visto que los trabajos que realizan propuestas para incluir la pronunciación de manera sistemática y natural en el aula de ELE son de diferente tipo y siguen un enfoque y una metodología diferentes. Como se ha señalado anteriormente, existe disparidad teórica en cuanto a la metodología a seguir a la hora de introducir e integrar la enseñanza de la pronunciación del español en el aula de ELE. Por este motivo, los investigadores se ven en la situación de tener que elegir un enfoque u otro, adaptarlo, y elaborar una propuesta de acuerdo con sus objetivos.

\section{Conclusión}

Al comienzo de este trabajo se ha señalado que existe una creencia generalizada entre el profesorado de español como lengua extranjera en torno a que los manuales de ELE suelen dejar de lado los aspectos relacionados con la enseñanza de la pronunciación del español. En este artículo se han analizado diferentes aspectos con el fin de descubrir cuál es el origen de esa creencia.

Como se ha podido comprobar, los creadores de manuales siguen las obras de referencia que, a su vez, se basan en las investigaciones en torno a la metodología de enseñanza de lenguas extranjeras y en las directrices de los diferentes métodos y enfoques. Por lo tanto, esta falta de atención a la pronunciación no se debe al tratamiento que los manuales hacen de ella, si no a una serie de aspectos que están intrínsicamente relacionados.

Si bien anteriormente se ha indicado que a Henry Sweet y Paul Passy les faltó concretar un auténtico método detallado, estructurado y basado en procedimientos científicos, parece que en la actualidad se tiende a obviar que se ha probado que incluir actividades basadas en el método verbo-tonal favorece la adquisición del sistema fonético de la lengua, y mejora la pronunciación de los estudiantes. No obstante, dado que no se ajusta a los métodos o enfoques más recientes, si se quiere seguir uno de ellos en concreto, como el enfoque comunicativo, sería conveniente realizar más propuestas de cómo integrar las actividades propias del método verbo-tonal en el enfoque comunicativo. No obstante, dado que, como indican algunos autores, nos encontramos en la época del posmétodo y del eclecticismo, no debería resultar complicado incluir actividades basadas en el método-verbo tonal en las aulas de ELE.

Por otro lado, sería interesante que los cursos de formación de profesores de ELE y de creación de materiales incluyan en sus programas la didáctica de la pronunciación del español como lengua extranjera, ya que, como se ha indicado previamente, la importancia de la pronunciación reside en que otorga inteligibilidad al texto oral. De esta manera los profesores tendrían los conocimientos y herramientas necesarios para integrarla en sus clases, y además podrían dejar a un lado los mitos y creencias que hay en torno a la enseñanza de la pronunciación.

También resultaría conveniente, como ya se hace en algunos casos, que a la hora de diseñar y crear materiales didácticos y manuales de ELE colaboraran expertos en didáctica de lenguas y expertos en fonética. De esta 
manera, se trabajarían estos contenidos de manera sistemática, y estarían en consonancia con las últimas investigaciones en este ámbito.

En conclusión, la situación actual de la enseñanza de la pronunciación del español en el aula de ELE se debe a un conjunto de factores, por lo que resultaría recomendable que haya un cambio global en todos ellos, de forma que haya un avance en la didáctica de este aspecto de la lengua, y que los estudiantes adquieran una buena competencia comunicativa.

\section{BIBLIOGRAFÍA}

BARTOLí Rigol, M. (2005): "La pronunciación en la clase de lenguas extranjeras", Phonica, vol. 1, pp. 1-27.

BARTOLÍ RIGOL, M. (2012): La pronunciación por tareas en la clase de E/LE. Tesis doctoral. Universitat de Barcelona.

http://diposit.ub.edu/dspace/bitstream/2445/54563/1/MBR TESIS.pdf

BARTOLí RIGOL, M. (2015): "Las actividades de pronunciación en los manuales de ELE”, CAUCE, no. 38, pp. 17-34.

CASANY, M. y LANDA, J. (2010): “Montañas, puentes y ríos: acercamiento a la pronunciación y a la entonación españolas en niveles iniciales", Foro de Profesores de E/LE, vol. 6.

Centro Virtual Cervantes (2008): Diccionario de términos clave de E/LE. https://cvc.cervantes.es/Ensenanza/biblioteca ele/diccio ele/defa ult.htm

Instituto Cervantes (2006): Plan curricular del Instituto Cervantes. Niveles de referencia para el español. Madrid: Biblioteca nueva.

CONSEJO DE EUROPA (2002): Marco común europeo de referencia para las lenguas: aprendizaje, enseñanza, evaluación. Madrid: Ministerio de Educación, Cultura y Deporte.

EzEIZA, J. (2009): “Analizar y comprender los materiales de enseñanza en perspectiva profesional: algunas claves para la formación del profesorado", Suplementos MarcoELE, vol. 9, pp. 1-58.

EzQUerRA, R. (1974): "Análisis de métodos para la enseñanza del español", Asociación Europea de Profesores de Español (AEPE), n. 11, pp. 3146.

GIL FERnÁNDEZ, J. (2007): Fonética para profesores de español: de la teoría a la práctica. Madrid: Arco Libros.

GonZÁLEZ SÁNCHEZ, M. (2016): Análisis metodológico de manuales de español para extranjeros: últimas aportaciones y perspectivas de futuro. Trabajo de final de máster. UNED. http://espacio.uned.es/fez/eserv/bibliuned:master-Filologia-FPESLMgonzalez/Gonzalez Sanchez Maria TFM.pdf

GOSPODARIČ, K. (2004): "La enseñanza de la pronunciación del español como lengua extranjera para eslovenohablantes: punto de partida", Verba Hispánica, n. 12, pp. 187-197.

IgARRETA Fernández, A. (2015): "La corrección de la pronunciación de los estudiantes sinohablantes en el aula de E/LE", Foro de Profesores de $E / L E$, n.ㅇ 11, pp. 189-196. 
IRUELA, A. (2004): Adquisición y enseñanza de la pronunciación en lenguas extranjeras. Tesis doctoral. Universitat de Barcelona. https://www.educacionyfp.gob.es/dam/jcr:efb6750d-661b-4237ac58-dfd08099069d/2009-bv-10-15iruela-pdf.pdf

Kumaravadivelu, B. (1994): "The Postmethod Condition: (E)merging Strategies for Second/Foreign Language Teaching" Tesol Quarterly, vol. 28, n.ㅇ․ pp. 27-48.

LLISTERRI, J. (2003): “La enseñanza de la pronunciación”, Revista del Instituto Cervantes en Italia, vol. 4, n.1, pp. 91-114.

MoLINA VIDAL, I. (2014): “Entonación, intención y relevancia. La importancia de la entonación y su enseñanza en el aula de ELE. Algunas propuestas didácticas", MarcoELE, n. 19.

NúÑez Delgado, M.a P. y RodríGuez GuerRero, B. (2017): “La comprensión lectora en ELE. Análisis de manuales de B1 con base en su tipología textual" Revista Internacional de Lenguas Extranjeras, n.6, pp. 3154.

Orta Gracia, Antonio (2009): "Creencias de los profesores acerca de la pronunciación y sus repercusiones en el aula", Phonica, vol. 5, pp. 4873.

Pérez Cano, I. (2019): "Las tareas de conciencia fonológica como recurso didáctico de comprensión auditiva en Español Lengua Extranjera (ELE)" Doblele, n. 5, pp. 66-84.

POCH OLIVÉ, D. (2004): "La pronunciación en la enseñanza del Español como Lengua Extranjera”, en RedELE Revista electrónica de didáctica, n.o 1.

Poch Olivé, D. y HARMEGIES, B. (2009): "Algunas cuestiones de pronunciación en la enseñanza del español como lengua extranjera", MarcoELE, n.으 8, pp. 105-110.

RENARD, R. (1979): Introduction á la méthode verbo-tonale de correction phonétique. Bruselas: Didier.

ŠIFRAR KALAN, M. (2017): "La enseñanza de la pronunciación en los manuales de ELE de A1-B2" Lingüística, vol. 57, n.o 1, pp. 313-330.

TIEDONG, Y. (2013): “Metodología y manuales en la enseñanza de español a sinohablantes", SinoELE, n. 8, pp. 17-41.

TORRERo DíAZ, R. (2020): "La enseñanza de la entonación en la clase de E/LE: propuesta de secuenciación didáctica" MarcoELE, n.은. 31. 